\title{
ON LIMITS OF SYSTEMS OF GROUPS
}

\section{H. B. GRIFFITHS}

In the course of some work in topology, it was necessary to prove Theorems 3.7 and 3.8 below. One of these concerns an inverse system of groups, the other a direct system, and it is easy to give a separate, ad hoc, proof of each. Both theorems are almost trivial. However, the theorems resemble each other so much that it seems of interest to find their common denominator. In this paper, we show that inverse and direct limits are special cases of a very general concept of "limit of a system of groups," and we derive the aforementioned theorems as special cases of a theorem in the general theory. The paper is therefore presented for the possible interest of the concept and method.

1. Definition of limit. Let $M$ be a set on which a relation $\Sigma$ is defined. For the present we impose no conditions on $\Sigma$ except that its definition be consistent. A group-system on $M$ is defined to be a pair $\langle G, R\rangle$ where to each $m \in M, G$ assigns a group $G(m)$ with unit element $1_{m}$, and whenever $m \Sigma n$ in $M, R$ assigns to the pair $(m, n)$ a subgroup $R_{m n}$ of the direct sum of $G(m)$ and $G(n)$. It will be convenient to tabulate the properties of $R_{m n}$ that we need; these hold be cause $R_{m n}$ is a group:

$$
(u, v) \in R_{m n} \cdot \Rightarrow \cdot\left(u^{-1}, v^{-1}\right) \in R_{m n}
$$

1.1

(ii) $\quad\left(1_{m}, 1_{n}\right) \in R_{m n}$;

(iii) $(u, v),(x, y) \in R_{m n} \cdot \Rightarrow \cdot(u x, v y) \in R_{m n}$.

If $X$ is a subset of $M$, we call a $\Sigma$-thread, or simply a thread, on $M$ any function $x$ which assigns to each $m \in X$ an element $x(m) \in G(m)$, and such that

$$
m \Sigma n \text { in } X \cdot \Rightarrow \cdot(x(m), x(n)) \in R_{m n} .
$$

Let $\Phi$ be a system of nonempty subsets of $M$ with the property:

$$
X, Y \in \Phi \cdot \Rightarrow \cdot X \cap Y \in \Phi,
$$

and define $\mathcal{P}$ to be the set of all pairs $(x, X)$ such that $X \in \Phi$ and $x$ is a thread on $X$. Of course, there is a functional relationship

$$
\odot=\odot(M, \Sigma, \Phi, G, R) \text {. }
$$

We shall now define a multiplication and then an equivalence relation $\Delta$ on $P$, such that $P / \Delta$ will be a group.

Received by the editors January 22, 1957 and, in revised form, April 26, 1957. 
Thus, let $(x, X) \in \mathcal{P}$, and define $(x, X)^{-1}$ to be $\left(x^{-1}, X\right)$, where for each $m \in X, x^{-1}(m)$ is defined to be $(x(m))^{-1}$. Then by (i) and (iv), $x^{-1}$ is a thread on $X$, and therefore $\left(x^{-1}, X\right) \in P$. Moreover, by (ii) and (iv), $\beta$ contains the element $\left(1_{X}, X\right)$, where for each $m \in X$,

$$
1_{X}(m)=1_{m} .
$$

Given $(x, X),(y, Y) \in \mathcal{P}$, we know by (v) that $X \cap Y \in \Phi$; and if $p \in X \cap Y$, then $x(p), y(p)$ are both defined in $G(p)$, as is their product $x(p) \cdot y(p)$. Define $x y(p)$ to be $x(p) \cdot y(p)$. Then $x y$ is a thread on $X \cap Y$ by (iii), since both $x$ and $y$ satisfy (iv) on $X \cap Y$. Therefore $(x y, X \cap Y) \in \odot$, and we define

$$
(x, X) \circ(y, Y)=(x y, X \cap Y) .
$$

We have thus turned $\rho$ into a multiplicative system, and the associativity of this multiplication follows from the associativity of set intersection and of each $G(m)$. Also,

$$
(x, X) \circ(x, X)^{-1}=(x, X) \circ\left(x^{-1}, X\right)=\left(x x^{-1}, X\right)=\left(1_{X}, X\right),
$$

and $(x, X) \circ\left(1_{X}, X\right)=\left(x 1_{X}, X\right)=\left(1_{X}, X\right) \circ(x, X)=(x, X)$ but in general $\left(1_{X}, X\right) \circ(y, Y) \neq(y, Y)$.

In order to obtain a group, we define an equivalence relation

$$
\Delta=\Delta(\odot)
$$

on $\odot$, by defining $(x, X) \Delta(y, Y)$ to mean that there exists $V \in \Phi$ such that

$$
x|V=y| V .
$$

Clearly $\Delta$ is reflexive and symmetric, and we now prove its transitivity. For if in addition $(y, Y) \Delta(z, Z)$, there exists $W \in \Phi$ such that $y|W=z| W$. Hence

$$
\begin{aligned}
z \mid V \cap W & =y \mid V \cap W \\
& =x \mid V \cap W \text { by }(\mathrm{x}) .
\end{aligned}
$$

But by (v), $V \cap W \in \Phi$, so that $(x, X) \Delta(z, Z)$, and $\Delta$ is transitive as asserted. This shows that $\Delta$ is a genuine equivalence relation on $P$.

It follows from (v) and the definition of multiplication that

$$
(x, X) \Delta(y, Y) \cdot \& \cdot\left(x^{\prime}, X^{\prime}\right) \Delta\left(y^{\prime}, Y^{\prime}\right)
$$

$$
: \Rightarrow:(x, X) \circ(y, Y) \Delta\left(x^{\prime}, X^{\prime}\right) \circ\left(y^{\prime}, Y^{\prime}\right) ;
$$

and hence for all $X, Y \in \Phi$, since

$$
\left(1_{X}, X\right) \Delta\left(1_{Y}, Y\right)
$$


then $\left(1_{X}, X\right) \circ(y, Y) \Delta\left(1_{Y}, Y\right) \circ(y, Y)=(y, Y) \Delta(y, Y) \circ\left(1_{X}, X\right)$. From the definitions it is also immediate that

$$
(x, X) \Delta(y, Y) \Rightarrow\left(x^{-1}, X\right) \Delta\left(y^{-1}, Y\right) .
$$

Hence, denoting by $\{(x, X)\}$ the equivalence class of $(x, X)$ in the quotient set $\rho / \Delta$, we can define

$$
\{(x, X)\} \cdot\{(y, Y)\}=\{(x y, X \cap Y)\}, \quad\{(x, X)\}^{-1}=\left\{\left(x^{-1}, X\right)\right\},
$$

and

$$
1=\left\{\left(1_{X}, X\right)\right\}
$$

without ambiguity, and thus turn $\rho / \Delta$ into a group, denoted by

A homomorphism

$$
\lim _{\Phi}\langle G, R\rangle_{(M, \Sigma)}
$$

$$
f:\langle G, R\rangle \rightarrow\langle H, S\rangle
$$

of one group-system on $M$ into another, is defined to be an assignment to each $m \in M$ of a homomorphism

$$
f_{m}: G(m) \rightarrow H(m)
$$

such that whenever $m \Sigma n$ in $M$,

$$
(u, v) \in R_{m n} \cdot \Rightarrow \cdot\left(f_{m} u, f_{n} v\right) \in S_{m n} .
$$

We shall define a homomorphism

$$
f_{*}: \lim _{\Phi}\langle G, R\rangle_{(M, \Sigma)} \rightarrow \lim _{\Phi}\langle H, S\rangle_{(M, \Sigma)}
$$

as follows. In the notation of (vi) and (ix), let

$$
\begin{gathered}
\odot_{K}=\rho(M, \Sigma, \Phi, K, T), \quad \text { where }(K, T)=(G, R) \text { or }(H, S), \\
\Delta_{K}=\Delta\left(\odot_{K}\right) .
\end{gathered}
$$

Given $(x, X) \in \odot_{G}$, define $(f x, X) \in \odot_{H}$ to be $(y, X)$, where for each $p \in X$,

$$
y(p)=f x(p)=f_{p}(x(p)) \in H(p) .
$$

By (xiii) and the fact that $x$ is a thread on $X, y$ is also a thread on $X$, and so $(y, X) \in \odot_{H}$ as asserted. Define $f^{\prime}: \odot_{G} \rightarrow \odot_{H}$ by $f^{\prime}(x, X)=(f x, X)$. By definition of the o operation in (vii), and the fact that each $f_{m}$ is a homomorphism, it follows that $f^{\prime}$ is itself a homomorphism. We assert (xvi)

$$
(x, X) \Delta_{G}(y, Y) \cdot \Rightarrow \cdot f^{\prime}(x, X) \Delta_{H} f^{\prime}(y, Y) .
$$


For, by definition there exists $V \in \Phi$ such that $x|V=y| V$, i.e., if $p \in V$, then $x(p)=y(p)$. Hence, by $(\mathrm{xv})$,

$$
f x(p)=f_{p}(x(p))=f_{p}(y(p))=f y(p)
$$

and so $f x|V=f y| V$. This proves (xvi). Hence, since $f^{\prime}: \mathcal{P}_{G} \rightarrow \odot_{H}$ is a homomorphism, so is the induced map $f_{*}: \odot_{G} / \Delta_{G} \rightarrow \mathcal{P}_{H} / \Delta_{H}$ defined by

$$
f_{*}\{(x, X)\}_{G}=\{(f x, X)\}_{H},
$$

where $\{\cdot\}_{K}$ denotes equivalence classes in $\odot_{K}$. This defines $f_{*}$ as required in (xiv).

2. Examples. We shall now consider examples of group-systems on $M$. In all of these, we assume the relation $\Sigma$ to be transitive, i.e.

$$
p \Sigma q \cdot \& \cdot q \Sigma r \text { in } M \cdot \Rightarrow \cdot p \Sigma r .
$$

$g h$-SYSTEMS. By a group-homomorphism (or gh) system $\langle A, a\rangle$ on $M$, we mean a pair which assigns to each $m \in M$ a group $A(m)$ and to each $m, n \in M$ with $m \Sigma n$, a homomorphism

$$
a_{m n}: A(m) \rightarrow A(n) .
$$

Then we obtain a group-system $\langle G, R\rangle$ on $M$ by taking $G=A$ and defining $R_{m n}=(R(a))_{m n}$ to be the graph of $a_{m n}$, i.e.

$$
(u, v) \in R_{m n} \cdot \Leftrightarrow \cdot v=a_{m n}(u) .
$$

Thus every gh-system $\langle A, a\rangle$ defines a group-system $\langle A, R(a)\rangle$ and we write

$$
\lim _{\Phi}\langle A, a\rangle_{(M, \Sigma)} \text { for } \lim _{\Phi}\langle A, R(a)\rangle_{(M, \Sigma)}
$$

In the applications, we shall require $\langle A, a\rangle$ to be transitive, i.e.

$$
m \Sigma n \Sigma p \text { in } M \cdot \Rightarrow \cdot a_{m p}=a_{n p} a_{m n} .
$$

If $\langle B, b\rangle$ is a second gh-system on $M$, and if $f:\langle A, R(a)\rangle \rightarrow\langle B, R(b)\rangle$ is a homomorphism in the sense of 1 (xii), then (ii) and 1 (xiii) imply that

$$
m \Sigma n \text { in } M \cdot \Rightarrow \cdot b_{m n} f_{m}=f_{n} a_{m n} .
$$

Conversely a set of homomorphisms $f_{m}: A(m) \rightarrow B(m)$ satisfying (iii) defines a homomorphism $f:\langle A, R(a)\rangle \rightarrow\langle B, R(b)\rangle$, and so we write

$$
f:\langle A, a\rangle \rightarrow\langle B, b\rangle
$$

to mean that for each $m \in M$, there is a homomorphism $f_{m}: A(m)$ $\rightarrow B(m)$ which satisfies (iii). 
The two most important known examples of gh-systems are the inverse and direct systems of groups and homomorphisms, to which we shall now devote the rest of this section.

Direct Limits. Let the relation $\Sigma$ on $M$ be transitive as above, and reflexive $(m \Sigma m$ for all $m \in M)$ and suppose that $\Sigma$ directs $M$, that is, if $m, n \in M$, there exists $p \in M$ satisfying $m \Sigma p$ and $n \Sigma p$. Let $\{G, g\}$ be a direct system of groups and homomorphisms on $M$; thus, for each $m \in M, G^{m}$ is a group, and whenever $p \Sigma q$ in $M$, there is a homomorphism $g_{p}^{q}: G^{p} \rightarrow G^{q}$, such that $g_{p}^{p}$ is the identity on $G^{p}$, and whenever $p \Sigma q \Sigma r$ in $M$,

$$
\stackrel{r}{g_{p}}=\stackrel{r}{g_{q}^{q} g_{p}}
$$

Thus, apart from notation, $\{G, g\}$ is a transitive gh-system $\langle G, g\rangle$ on $(M, \Sigma)$. Denote the direct limit of $\{G, g\}$ by

$$
G^{\infty}=\operatorname{Dlim}\{G, g\} \text {. }
$$

A subset $X \subseteq M$ will be called "saturated in $M$ " whenever $X$ is nonempty and

$$
x \Sigma m \& x \in X \cdot \Rightarrow \cdot m \in X
$$

Hence, if $\Phi$ is the family of all saturated subsets of $M$, then $\Phi$ satisfies 1 (v) since $M$ is directed by $\Sigma$.

2.1. Theorem. Dlim $\{G, g\} \approx \lim _{\Phi}\langle G, g\rangle_{(M, \leqq)}$.

Proof. We recall that $\operatorname{Dlim}\{G, g\}$ is the set of equivalence classes $^{1}\left[x^{m}\right]$ in $\bigcup_{m} G^{m}$, where, given $x^{m} \in G^{m}$ and $x^{n} \in G^{n}$, then

$$
x^{m} \equiv x^{n} \text { or }\left[x^{m}\right]=\left[x^{n}\right]
$$

if and only if there exists $p \in M$ such that $m, n \Sigma p$ and

$$
g_{m}^{p} x^{m}=g_{n}^{p} x^{n} \text {. }
$$

We assert that if $(x, X),(y, Y)$ are two $\Delta$-equivalent elements in $\mathcal{P}=\mathcal{P}(M, \Sigma, \Phi, G, R(g)$ ) (see $1(\mathrm{vi})$ ) then all the values $x(m), y(n)$ of $x$ and $y$ lie in the same element of $\operatorname{Dlim}\{G, g\}=G^{\infty}$. For, by $1(\mathrm{x})$, there exists $V \in \Phi$ such that $x|V=y| V$; and since $M$ is directed and $V$ saturated, there exists to each $m \in X, n \in Y$, an element $p \in V$ such that $m, n \Sigma p$. Since $x, y$ are threads, then we have from $m \Sigma p, n \Sigma p$ that, respectively

$$
x(p)=\stackrel{p}{g_{m}} x(m), \quad y(p)=g_{n}^{p} y(n) ;
$$

${ }^{1}$ We have no cause here to consider powers of $x$; the $m$ is to be read as a label, not power. 
but $x(p)=y(p)$ since $p \in V$. Therefore (i) is satisfied, and so $x(m)$ $\equiv y(n)$, i.e. $[x(m)]=[y(n)]$ as asserted.

Hence, to each $\alpha=\{(x, X)\} \in \mathcal{Q} / \Delta$, we assign the unique class $[x(m)] \in G^{\infty},(m \in X)$, and denote it by $\theta \alpha$. It is easily verified that $\theta$ is a homomorphism, and we shall prove that $\theta$ is an isomorphism.

(a) $\theta$ is onto. For let $\alpha \in G^{\infty}$, and choose $x^{m} \in \alpha$. Define $X_{m}$ to be the set of all $p \in M$ such that $m \Sigma p$. Since $\Sigma$ is transitive and directs $M$, then $X_{m}$ is saturated, and so $X_{m} \in \Phi$. Each $p \in X_{m}$ satisfies $m \Sigma p$, so that we define $x(p)$ to be $g_{m}^{p} x^{m}$; and $x$ is a thread on $X_{m}$ because $\langle G, g\rangle$ is transitive. Since $g_{m}^{m}=$ identity, then $x(m)=x^{m}$, so that $[x(p)]=[x(m)]=\alpha$ for all $p \in X$. Hence $\alpha=\theta\left(x, X_{m}\right)$, which proves that $\theta$ is onto, as asserted.

(b) $\theta$ is univalent. For, suppose $\theta\{(x, X)\}=1$, the unit of $G^{\infty}$. Then by (i), if $m \in X$ there exists $p \in M$ such that $m \Sigma p$ and $g_{m}^{p} x(m)=1_{p}$, the unit of $G^{p}$. But $p \in X$ also, since $X$ is saturated, so that $x(p)$ is defined, and is $g_{m}^{p} x(m)$ since $x$ is a thread on $X$. Thus $x(p)=1_{p}$ for all $p$ in the set $X_{m}$ (defined in (a)); so that $x \mid X_{m}=1_{X_{m}}=e_{m}$, say. Hence $(x, X) \Delta\left(e_{m}, X_{m}\right)$ since $X_{m} \in \Phi$, and so

$$
\{(x, X)\}=\left\{\left(e_{m}, X_{m}\right)\right\}=\text { unit of } \odot / \Delta,
$$

whence $\theta$ is univalent, as asserted.

That $\theta$ is an isomorphism follows from (a) and (b), and the proof of the theorem is complete.

Inverse Limits. Let $M$ be directed by $\Sigma$ as before, and let $(H, h)$ be an inverse system of groups and homomorphisms on $M$; thus, for each $m \in M, H_{m}$ is a group, and whenever $m \Sigma n$ in $M$ there is a homomorphism $h_{m}^{n}: H_{n} \rightarrow H_{m}$, such that $h_{m}^{m}$ is the identity on $H_{n}$, and if $m \Sigma n \Sigma p$ in $M$ then $h_{m}^{p}=h_{m}^{n} h_{n}^{p}$. To obtain a gh-system we have to reverse the direction of $\Sigma$; let $m \Sigma^{-1} n$ mean that $n \Sigma m$ in $M$. Then, writing $h_{p m}$ for $h_{m}^{p},(H, h)$ is a transitive gh-system $\langle H, h\rangle$ on $\left(M, \Sigma^{-1}\right)$. We denote the inverse limit of $(H, h)$ by

$$
G_{\infty}=\mathrm{I} \lim (H, h)_{M} .
$$

Let $\Psi$ be the system whose only element is $M$. Then trivially $\Psi$ satisfies $1(\mathrm{v})$.

2.2. Theorem. $\operatorname{Ilim}(H, h)_{M} \approx \lim _{\Psi}\langle H, h\rangle_{\left(M, \Sigma^{-1}\right)}$.

Proof. By definition, each element of $G_{\infty}$ is a $\Sigma^{-1}$-thread over $M$, in the sense of 1(iv). Hence, if in the sense of 1(vi),

$$
\odot=\odot\left(M, \Sigma^{-1}, \Psi, H, R(h)\right)
$$

then $\rho$ is identical with $G_{\infty}$; while by definition of $\Psi$ the relation 
$\Delta=\Delta(\odot)$ of 1 (ix) is the relation of equality. Hence $P / \Delta=\odot$, which we have seen to be $G_{\infty}$. Thus

$$
\operatorname{Ilim}(H, h)_{M}=\rho=\rho / \Delta=\lim _{\Psi}\langle H, h\rangle_{\left(M, \Sigma^{-1}\right)},
$$

which proves the theorem.

As in 2.1 , let $\Phi$ be the system of saturated subsets of $(M, \Sigma)$. Then a second way of expressing $G_{\infty}$ in the form 2.2 is given by

2.3. Theorem. $\operatorname{Ilim}(H, h)_{M} \approx \lim _{\Phi}\langle H, h\rangle_{\left(M, \Sigma^{-1}\right)}$.

Proof. Let $\mathcal{P}=\mathcal{P}\left(M, \Sigma^{-1}, \Phi, H, R(h)\right)$ in the sense of 1 (vi). Given any $(x, X) \in P, x$ is by definition a $\Sigma^{-1}$-thread on $X$; and since $M$ is directed, $x$ has a unique extension to $x_{*}$, a $\Sigma^{-1}$-thread on $M$, so that $x_{*}|X=x| X=x$. Now $M$ is saturated in $M$, and so $M \in \Phi$. Hence $\left(x_{*}, M\right)$ lies in the same $\Delta$-equivalence class of $\rho$, where $\Delta=\Delta(\rho)$ as in 1 (ix). Define $\psi: \rho / \Delta \rightarrow G_{\infty}$ by $\psi\{(x, X)\}=x_{*}$. It now follows easily that $\psi$ is an isomorphism, and so

$$
\operatorname{Ilim}(H, h)_{M}=G_{\infty} \approx \mathcal{P} / \Delta=\lim _{\Phi}\langle H, h\rangle_{\left(M, \Sigma^{\prime}\right)}
$$

as required.

3. Isomorphic gh-systems. Now let us consider gh-systems in more detail. Let the relation $\Sigma$ on $M$ be transitive but not necessarily reflexive; we do not require $\Sigma$ to direct $M$. Let $\Phi$ be a family of subsets of $M$ satisfying $1(\mathrm{v})$. Given two gh-systems $\langle A, a\rangle,\langle B, b\rangle$ on $(M, \Sigma)$ let

$$
f:\langle A, a\rangle \rightarrow\langle B, b\rangle .
$$

Thus, as in 2(v) and 1 (xiv) there is an induced homomorphism

$$
f_{*}: \lim _{\Phi}\langle A, a\rangle_{(M, \Sigma)} \rightarrow \lim _{\Phi}\langle B, b\rangle_{(M, \Sigma)},
$$

or briefly

$$
f_{*}: A_{*} \rightarrow B_{*} \text {. }
$$

We shall now discuss a set of sufficient conditions, arising in topology, for $f_{*}$ to be an isomorphism. Suppose that the following condition holds:

3.1. If $m \Sigma n$ in $M$, there is a homomorphism

$$
\psi_{m n}: B(m) \rightarrow A(n) .
$$

In the applications we have in mind, one or both of the following conditions is also satisfied: 
(a) $m \Sigma n$ in $M \cdot \Rightarrow \cdot a_{m n}=\psi_{m n} f_{m}$.

(b) $m \Sigma n$ in $M \cdot \Rightarrow \cdot b_{m n}=f_{n} \psi_{m n}$.

3.3. Lemma. If $m \Sigma n \Sigma p$ in $M$ and if the $\psi$ 's satisfy both 3.2(a) and (b), then

$$
a_{n p} \psi_{m n}=\psi_{n p} b_{m n} .
$$

Proof. We can draw the following diagram:

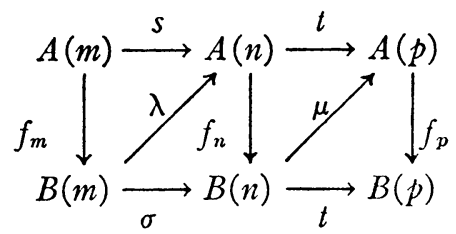

where for typographical reasons we have designated the homomorphisms as shown. We have to prove

$$
t \lambda=\mu \sigma,
$$

so that by 2 (iv) the diagram will then be everywhere commutative. But

$$
\begin{aligned}
t \lambda & =\mu f_{n} \lambda \\
& =\mu \sigma
\end{aligned}
$$

as required.

Now consider the following condition on $\Phi$.

3.4. If $X \in \Phi$ there exists $Z \in \Phi$ such that $Z \subseteq X$, and if $p \in Z$, there exists $m=\mu(p, X) \in X$ such that

$$
m \Sigma p .
$$

3.5. Theorem. If the $\psi$ 's of 3.1 satisfy $3.2(\mathrm{a})$, and if $\Phi$ satisfies 3.4(a), then in (ii) above, $f_{*}$ is univalent.

Proof. By the definitions, $A_{*}$ is of the form $\odot_{A} / \Delta_{A}$, and if $\alpha$ $=\{(x, X)\} \in A_{*}$ then

$$
f_{* \alpha}=\{(f x, X)\}
$$

where for all $p \in X$,

$$
f x(p)=f_{p}(x(p)) \in B(p) .
$$

Now if $f_{*} \alpha=1_{B}$ (the unit of $B_{*}$ ), then there exists $V \in \Phi$ such that $(f x, X) \Delta_{B}\left(1_{B, V}, V\right)$, i.e. if $v \in V$, then $f x(v)=1_{B v}$ (the unit of $\left.B(v)\right)$. By 3.4(a), there exists $W \subseteq X \cap V$ in $\Phi$, and if $w \in W$ there exists 
$m=\mu(w, X \cap V) \in X$ such that $m \Sigma w$. Hence $\psi_{m w}$ and $x(m)$ are defined, and since $\psi_{m n}$ is a homomorphism, then

$$
\begin{aligned}
1_{A w}=\psi_{m w} 1_{B m}=\psi_{m w} f x(m) & =\psi_{m w} f_{m}(x(m)) \\
& =a_{m w}(x(m)) \\
& =x(w)
\end{aligned}
$$

since $x$ is a thread on $X$. This proves that for all $w \in W, x(w)=1_{A w}$, i.e. $x\left|W=1_{A w}\right| W$, so that $(x, X) \Delta_{A}\left(1_{A W}, W\right)$. Therefore $\{(x, X)\}$ $=\alpha=1_{A}$, which proves $f_{*}$ to be univalent, as required.

In order to prove $f_{*}$ to be onto, we have to make extra assumptions. First, let $\Sigma^{\prime}$ be a second transitive order-relation on $M$, which is weaker than $\Sigma$; that is, if $m \Sigma n$ in $M$, then also $m \Sigma^{\prime} n$. Suppose also that $\langle A, a\rangle,\langle B, b\rangle$ are gh-systems over $\left(M, \Sigma^{\prime}\right)$ (so that if $m \Sigma^{\prime} n$ in $M$, then $a_{m n}: A_{m} \rightarrow A_{n}$ and $b_{m n}: B_{m} \rightarrow B_{n}$ are defined). Finally suppose that condition $3.4(\mathrm{a})$ holds with the addition

3.4(b) $\quad p \Sigma q$ in $Z \cdot \Rightarrow \cdot \mu(p, X) \Sigma^{\prime} \mu(q, X)$ or $\mu(p, X)=\mu(q, X)$.

3.6. THEOREM. If the $\psi$ 's of 3.1 satisfy $3.2(\mathrm{a})$ and (b) whenever $m \Sigma^{\prime} n$ in $M$, and if $\Phi$ satisfies 3.4(a) and (b), then $f_{*}$ is onto.

Proof. Let $\beta \in B_{*}$ and let $(y, Y) \in \beta$, so that for each $p \in Y$, $y(p) \in B(p)$. Since $\Phi$ satisfies $3.4(\mathrm{a})$, there exists $W \in \Phi$ such that $W \subseteq Y$, and if $w \in W$ there exists $m=\mu(w, Y) \in Y$ such that $m \Sigma w$. Hence $m \Sigma^{\prime} w$, and so $\psi_{m w}$ is defined, as well as $y(m), y(w)$; define

$$
x(w)=\psi_{m w} y(m) .
$$

Then

$$
\begin{aligned}
f x(w)=f_{w}(x(w)) & =f_{w} \psi_{m w} y(m) \\
& =b_{m w} y(m) \\
& =y(w)
\end{aligned}
$$

since $y$ is a $\Sigma$-thread on $Y$. Hence, if we can prove that $x$ is a $\Sigma$ thread on $W$, then we shall have shown that

$$
\begin{aligned}
f_{*}\{(x, W)\}=\{(f x, W)\} & =\{(y \mid W, W)\} \\
& =\{(y, Y)\} \text { since } W \in \Phi,
\end{aligned}
$$

and therefore that $f_{*}$ is onto.

To prove that $x$ is a thread on $W$, let $w \Sigma v$ in $W$. We have to show that

$$
a_{w v} x(w)=x(v)
$$


i.e. that

$$
a_{w v} \psi_{m w} y(m)=\psi_{n v} y(n)
$$

where

$$
m=\mu(w, Y), \quad n=\mu(v, Y) .
$$

Now

$$
\begin{aligned}
a_{w v} \psi_{m w} y(m) & =\psi_{w v} b_{m w} y(m) \\
& =\psi_{w v} y(w)
\end{aligned}
$$

since $y$ is a thread on $Y$. But for any $q \in M$ such that $m \Sigma^{\prime} q \Sigma^{\prime} v$, the homomorphism $a_{m v}$, for example, is defined; and so we have

$$
\begin{aligned}
\psi_{m v} y(m) & =\psi_{m v} f_{m}(x(m)) & \text { by (i) above, } \\
& =a_{m v} x(m) & \text { by } 3.2(\mathrm{a}) ;
\end{aligned}
$$

while

$$
\begin{aligned}
\psi_{q v} y(q) & =\psi_{q v} b_{m q} y(m) \\
& =\psi_{q v} b_{m q} f_{m}(x(m)) \\
& =\psi_{q v} f_{q} a_{m q} x(m) \\
& =a_{q v} a_{m q} x(m) \\
& =a_{m v} x(m) .
\end{aligned}
$$

By 3.4(b), $w \Sigma v$ implies $m \Sigma^{\prime} n$ (from (iv)) or else $m=n$; and so the relations

$$
m \Sigma w \Sigma v ; \quad m \Sigma^{\prime} n \Sigma v, \quad \text { or } m=n,
$$

enable us to put $q=w, n$ in the above, since $\Sigma$ is stronger than $\Sigma^{\prime}$, to get

$$
\psi_{m v} y(m)=\psi_{w v} y(w)=\psi_{n v} y(n) .
$$

Combining this with (v) gives (iii); and the proof is complete.

REMARK. The correspondence $\pi:\{(y, Y)\} \rightarrow\{(x, W)\}$, constructed at the beginning of the proof of the theorem, is defined only in terms of the $\psi$ 's and the functions $\mu$ and $Y \rightarrow W$; although we used $f$ to verify that $\pi\{(y, W)\}$ is a genuine element of $B_{*}$. And since we proved that $f_{*} \pi$ is the identity on $B_{*}$, then $\pi=f_{*}^{-1}$ since $f_{*}$ is an isomorphism. Therefore $\pi: B_{*} \rightarrow A_{*}$ is an isomorphism, and so we have the

Corollary. If $f:\langle A, a\rangle \rightarrow\langle B, b\rangle$ is any homomorphism satisfying $3.2(\mathrm{a})$ and (b) for the fixed set of $\psi$ 's, then $f_{*}$ is the unique isomorphism $\pi^{-1}$, which depends only on the $\psi$ 's. 
Let us apply these results to the special cases of direct and inverse limits. In each case, we take $M$ to be directed by the reflexive and transitive relation $\leqq$; and shall have to assume that $M$ is strongly directed by $\leqq$, i.e. that $M$ is directed by < (equality excluded), otherwise the theorems below will be obviously false. Considering direct limits first, we take $\Phi$ as in 2.1 to be the family of saturated subsets of $M$. If $U \in \Phi$, choose a definite element

$$
j=\eta(U) \in U
$$

- as we can since $U$ is by definition nonempty - and define $Z=Z(U)$ to be the set of all $m \in M$ such that $u<m$. Then $Z$ is nonempty since $M$ is strongly directed; and $Z$ is saturated since $\leqq$ is transitive. Hence $Z \in \Phi$ and $Z \subseteq U$. Define

$$
\sigma(m, U)=u
$$

for all $m \in Z$. Thus by definition,

$$
\sigma(m, U)<m,
$$

and

$$
m, n \in Z \cdot \Rightarrow: \sigma(m, U)=\sigma(n, U) .
$$

Therefore $\Phi$ satisfies 3.4 (b) with $\mu, \Sigma, \Sigma^{\prime}$ taken to be $\sigma,<$, and $<$ respectively. Hence, coupling 2.1 with 3.5 and 3.6 gives

3.7. Theorem. Let $(M, \leqq)$ be directed by $<$, and let $\{A, a\},\{B, b\}$ be direct systems of groups and homomorphisms on $(M, \leqq)$. Let $f:\{A, a\} \rightarrow\{B, b\}$ be a homomorphism, inducing

$$
f^{\infty}: \operatorname{Dlim}\{A, a\} \rightarrow \operatorname{Dlim}\{B, b\},
$$

and suppose that whenever $m<n$ in $M$, there exists a homomorphism

$$
\psi_{m n}: B^{m} \rightarrow A^{n} \text {. }
$$

If $\psi_{m n} f_{n}=a_{m}^{n}$ then $f^{\infty}$ is univalent; and if in addition $f_{m} \psi_{m n}=b_{m}^{n}$ then $f^{\infty}$ is onto and $f^{\infty}=g^{\infty}$ for any $g$ satisfying the same data as $f$ above.

For inverse limits, let $(M, \leqq)$ be strongly directed as above, let $\Phi$ consist of the single set $M$, and recall from 2.2 that if $(A, a)$ is an inverse system of groups and homomorphisms on $(M, \leqq)$ then

$$
\operatorname{Ilim}(A, a) \approx \lim _{\Phi}\langle A, a\rangle_{(M, \geq)} .
$$

To apply 3.5 and 3.6 we shall take $\Sigma^{\prime}$ to be $>$. Since $M$ is directed by $<$, then to each $m \in M$ there exists $n \in U$ such that $m<n$, i.e. $n \Sigma^{\prime} m$; choose such an $n$ and define it to be $\tau(m)$. Then define a new 
order relation $\Sigma$ on $M$, by $p \Sigma m$ whenever $\tau(m) \leqq p$. Clearly $\Sigma$ is transitive and stronger than $\Sigma^{\prime}$; and $3.4(\mathrm{a})$ and (b) hold with $\mu(m, X)$ $=\tau(m)$ and $Z=M($ since $\Phi=\{M\})$.

Now, in the isomorphism (i) above, it is easily verified that the right-hand side is $\lim _{\Phi}\langle A, a\rangle_{(M, \Sigma)}$, and similarly for $(B, b)$. Hence applying 3.5 and 3.6 gives

3.8. Theorem. Let $(m, \leqq)$ be strongly directed by $<$, and let $(A, a)$, $(B, b)$ be inverse systems of groups and homomorphisms on $(M, \leqq)$. Let $f:(A, a) \rightarrow(B, b)$ be a homomorphism, inducing

$$
f_{\infty}: \operatorname{Ilim}(A, a) \rightarrow \operatorname{Ilim}(B, b)
$$

and suppose that whenever $n>m$ in $M$ there exists a homomorphism

$$
\psi_{n m}: B(n) \rightarrow A(m) .
$$

If $\psi_{n m} f_{n}=a_{m}^{n}$, then $f_{\infty}$ is univalent. If, in addition, $f_{m} \psi_{n m}=b_{m}^{n}$ then $f_{\infty}$ is onto, and $f_{\infty}=g_{\infty}$ for any $g$ satisfying the same data as $f$ above.

REMARK. It would be desirable to have in the general case an analogue of the "cofinality theorems" for inverse and direct limits, to enable one to take a simpler system than $(M, \Sigma, \Phi)$ when computing a limit of the form $\lim _{\Phi}\langle G, R\rangle_{(M, \Sigma)}$. One can think of various sets of conditions which give such an analogous theorem, but owing to the generality required no significant and elegant set seems to present itself. Rather than burdening the reader with possibly artificial results, it seems better to wait until more examples of these general limits occur in practice for a natural theorem may then become obvious.

Institute for Advanced Study 